\title{
DE FINETTI'S OPTIMAL DIVIDENDS PROBLEM WITH AN AFFINE PENALTY FUNCTION AT RUIN
}

\author{
RONNIE L. LOEFFEN AND JEAN-FRANÇOIS RENAUD
}

\begin{abstract}
In a Lévy insurance risk model, under the assumption that the tail of the Lévy measure is log-convex, we show that either a horizontal barrier strategy or the take-the-money-and-run strategy maximizes, among all admissible strategies, the dividend payments subject to an affine penalty function at ruin. As a key step for the proof, we prove that, under the aforementioned condition on the jump measure, the scale function of the spectrally negative Lévy process has a log-convex derivative.
\end{abstract}

\section{INTRODUCTION AND MAIN RESUlT}

In 1957, Bruno de Finetti [14 formulated the optimal dividends problem: it is the quest for the dividend policy maximizing the expected present value of the dividend payments made by an insurance company. Nowadays, this control problem is known as de Finetti's optimal dividends problem and still attracts a lot of research interest. Another burgeoning research topic in insurance mathematics is the analysis of the so-called Gerber-Shiu functions. As it is now well known, these functions, also called expected discounted penalty functions, were first introduced by Gerber and Shiu [18]. In this paper, we formulate and solve an optimal dividends problem taking into account the time of ruin and the deficit at ruin using a particular GerberShiu function.

1.1. Lévy insurance risk processes. The compound Poisson risk model describes the (free) surplus of an insurance company/portfolio using a compound Poisson process with drift, that is

$$
X_{t}-X_{0}=c t-\sum_{i=1}^{N_{t}} C_{i},
$$

where $c>0$ denotes the constant premium intensity, $N=\left(N_{t}\right)_{t \geq 0}$ is a Poisson process and the $C_{i}$ 's are (strictly) positive independent and identically distributed random variables representing the claim amounts. In this model, the random variable $\sum_{i=1}^{N_{t}} C_{i}$ represents the aggregate claim payments

Date: September 21, 2009.

2000 Mathematics Subject Classification. 60J99, 93E20, 60G51.

Key words and phrases. Insurance risk theory, optimal dividends, deficit at ruin, Gerber-Shiu functions, Lévy processes, stochastic control, log-convexity. 
made up to time $t$. One way to generalize this risk process is to consider a spectrally negative Lévy process $X=\left(X_{t}\right)_{t \geq 0}$, that is a Lévy process with no positive jumps. This process is defined on a filtered probability space $\left(\Omega, \mathcal{F},\left(\mathcal{F}_{t}\right)_{t \geq 0}, \mathbb{P}\right)$ and its law when $X_{0}=x$ is denoted by $\mathbb{P}_{x}$ (and the corresponding expectation by $\mathbb{E}_{x}$ ). For short, we write $\mathbb{P}$ and $\mathbb{E}$ when $X_{0}=0$. The process $X$ has independent and stationary increments, and has càdlàg paths (right-continuous with left limits). We assume that the filtration satisfies les conditions habituelles (usual conditions), namely right-continuity and completeness. To avoid trivialities, it is implicitly assumed that $X$ does not have monotone sample paths, that is $X$ is not a negative subordinator, as for example a compound Poisson process with a negative drift, or just a deterministic drift. In the actuarial literature, $X$ is often called a Lévy insurance risk process; see [9, 22, for more details.

As the Lévy process $X$ has no positive jumps, its Laplace transform exists and is given by

for $\theta \geq 0$ and $t \geq 0$, where

$$
\mathbb{E}\left[\mathrm{e}^{\theta X_{t}}\right]=\mathrm{e}^{t \psi(\theta)}
$$

$$
\psi(\theta)=\gamma \theta+\frac{1}{2} \sigma^{2} \theta^{2}-\int_{0}^{\infty}\left(1-\mathrm{e}^{-\theta z}-\theta z \mathbb{I}_{(0,1]}(z)\right) \nu(\mathrm{d} z)
$$

for $\gamma \in \mathbb{R}$ and $\sigma \geq 0$, and where $\nu$ is a measure on $(0, \infty)$ such that

$$
\int_{0}^{\infty}\left(1 \wedge z^{2}\right) \nu(\mathrm{d} z)<\infty
$$

The measure $\nu$ is called the Lévy measure of $X$, while $(\gamma, \sigma, \nu)$ is referred to as the Lévy triplet of $X$. Note that the compound Poisson risk process defined in (1) corresponds to the case $\gamma=c-\int_{0}^{1} z \nu(\mathrm{d} z), \sigma=0$ and $\nu(\mathrm{d} z)=\lambda F(\mathrm{~d} z)$, where $\lambda$ is the jump intensity of $N$ and where $F$ is the distribution of the $C_{i}$ 's, while a Brownian motion risk process corresponds to the case $\sigma>0$ and $\nu(\mathrm{d} z) \equiv 0$. It is well known that $X$ has paths of bounded variation if and only if $\sigma=0$ and $\int_{0}^{1} z \nu(\mathrm{d} z)<\infty$. Finally, note that the net profit condition for a general Lévy insurance risk process is given by

$$
\mathbb{E}\left[X_{1}\right]=\psi^{\prime}(0+)=\gamma-\int_{1}^{\infty} z \nu(\mathrm{d} z)>0
$$

which agrees with the classical formulation if $X$ is a compound Poisson process with drift, as in the Cramér-Lundberg model. However, in what follows, this condition will not be assumed.

1.2. Problem formulation. We now state the control problem considered in this paper. A dividend strategy $\pi$ consists of a nondecreasing, leftcontinuous and adapted process $L^{\pi}=\left(L_{t}^{\pi}\right)_{t \geq 0}$, where $L_{t}^{\pi}$ represents the cumulative amount of dividends paid until time $t$ under this strategy, and such that $L_{0}^{\pi}=0$. A lump sum dividend payment is then represented by a jump of the process $L^{\pi}$. For a given dividend policy $\pi$, the corresponding controlled reserve process $U^{\pi}=\left(U_{t}^{\pi}\right)_{t \geq 0}$ is defined by $U_{t}^{\pi}=X_{t}-L_{t}^{\pi}$ and the 
ruin time is then given by $\sigma^{\pi}=\inf \left\{t \geq 0: U_{t}^{\pi}<0\right\}$. A strategy $\pi$ is said to be admissible if $L_{t+}^{\pi}-L_{t}^{\pi} \leq U_{t}^{\pi}$, for all $t<\sigma^{\pi}$, and if $L_{\sigma^{\pi}+}^{\pi}-L_{\sigma^{\pi}}^{\pi}=0$ when $\sigma^{\pi}<\infty$. In other words, the company does not make a lump sum dividend payment which is larger than its current surplus. The set of admissible dividend strategies will be denoted by $\Pi$.

Let $q>0$ represent the force of interest. In this paper, the value function of a dividend strategy $\pi$ is defined by

$$
v_{\pi}(x)=\mathbb{E}_{x}\left[\int_{0}^{\sigma^{\pi}} \mathrm{e}^{-q t} \mathrm{~d} L_{t}^{\pi}+\mathrm{e}^{-q \sigma^{\pi}} P\left(U_{\sigma^{\pi}}^{\pi}\right) \mathbb{I}_{\left\{\sigma^{\pi}<\infty\right\}}\right],
$$

where $P(y)=S+K y$, for $S \in \mathbb{R}$ and $K \in(0,1]$. By definition, it follows that $v_{\pi}(x)=S+K x$ for $x<0$. The control problem is to find the optimal value function $v_{*}$ defined by

$$
v_{*}(x)=\sup _{\pi \in \Pi} v_{\pi}(x)
$$

and an optimal strategy $\pi_{*} \in \Pi$ such that

$$
v_{\pi_{*}}(x)=v_{*}(x),
$$

for any starting capital $x \geq 0$.

Note that the function $\bar{P}$ is a (degenerate) penalty function and that

$$
\mathbb{E}_{x}\left[\mathrm{e}^{-q \sigma^{\pi}} P\left(U_{\sigma^{\pi}}^{\pi}\right) \mathbb{I}_{\left\{\sigma^{\pi}<\infty\right\}}\right]
$$

is the corresponding (degenerate) Gerber-Shiu function. Therefore, we refer to this problem as de Finetti's optimal dividends problem with a Gerber-Shiu function.

This specific choice of penalty function, namely $P(y)=S+K y$, can be motivated as follows. As $K \in(0,1]$, the shareholders are held responsible to cover (part of) the deficit at ruin and, if $S<0$, early ruin is penalized, while, if $S>0$, shareholders will benefit from a liquidation value at ruin. However, this modification of the classical optimal dividend problem is not completely new. First, it should be noted that the 'original' de Finetti's optimal dividends problem corresponds to $S=K=0$, i.e., when the penalty function $P$ is identically zero. This case has been extensively studied. The case $S<0$ and $K=0$ was studied by Thonhauser and Albrecher [33] for the compound Poisson risk process and the Brownian motion risk process, while Boguslavskaya [11], and Shreve, Lehoczky and Gaver [32, considered the case $S \in \mathbb{R}$ and $K=0$ in a Brownian motion and diffusion setting, respectively. Finally, the case $S \in \mathbb{R}$ and $K=0$ has been analyzed by Loeffen [25] for spectrally negative Lévy processes. Dickson and Waters [15, Gerber, Shiu and Smith [19, and Gerber, Lin and Yang [17] looked at related value functions and problems in the compound Poisson setting; the case $S=0$ and $K=1$ was discussed in [15, 19] and a numerical study, for penalty functions taking the form of a polynomial of degree less than or equal to 2, was carried out in [17. In the Lévy setting, Avram, Palmowski and Pistorius [7] studied a model where capital is injected (repeatedly) to 
keep the surplus process afloat (for the compound Poisson case, see also the paper of Kulenko and Schmidli [21]). This is close in spirit to our problem as it considers the fact that the surplus process can go negative eventually and that the shareholders should then be held responsible to cover the deficit. Consequently, the objective function under study is closely related to the so-called Dickson and Waters modifications [15]. For more background and literature coverage on dividend strategies and optimal dividends problems in risk theory, we refer the reader to the reviews of Avanzi [5] and Albrecher and Thonhauser [1] respectively.

1.3. Main results. Before stating our main results, we introduce $\pi_{b}$, the horizontal barrier strategy at level $b \geq 0$. Under this strategy, $\sigma^{b}$ denotes the time of ruin, which is then almost surely finite, and the controlled risk process is denoted by $U_{t}^{b}=X_{t}-L_{t}^{b}$, with $L_{0}^{b}=0$ and $L_{t}^{b}=\left(\sup _{0 \leq s \leq t} X_{s}-b\right)^{+}$, for all $t>0$. Note that $\pi_{b} \in \Pi$ and, if $X_{0}=x>b, L_{0+}^{b}=x-b$.

As ruin occurs when the controlled risk process is strictly below 0 , the barrier strategy at 0 will cause immediate ruin for all but one risk process, namely the compound Poisson risk process. Therefore, we define the take-the-money-and-run strategy as the strategy where all the surplus is immediately paid out, that is at time 0 , and then ruin is forced. We denote this admissible strategy by $\pi_{\text {run. }}$. Clearly, we have that $v_{\text {run }}(x)=x+S$, for all $x \geq 0$.

Recall that the tail of the Lévy measure is the function $x \mapsto \nu(x, \infty)$, where $x \in(0, \infty)$ and that a function $f:(0, \infty) \rightarrow[0, \infty)$ is said to be $\log$-convex (log-concave) if $\log (f)$ is convex (concave) on $(0, \infty)$.

Theorem 1.1. Let $X$ be a Lévy insurance risk process with Lévy triplet $(\gamma, \sigma, \nu)$ and let $c=\gamma+\int_{0}^{1} z \nu(\mathrm{d} z)$. Recall that $q>0,0<K \leq 1$ and $S \in \mathbb{R}$. If the tail of the Lévy measure is log-convex, then the following hold:

(i) Suppose $\psi^{\prime}(0+)>-\infty$. If $\sigma>0$, or $\nu(0, \infty)=\infty$, or $\nu(0, \infty)<\infty$ and $c \geq K \int_{0}^{\infty} z \nu(\mathrm{d} z)+S q$, then an optimal strategy for the control problem is formed by a barrier strategy.

(ii) Suppose $\psi^{\prime}(0+)>-\infty$. If $\sigma=0$ and $\nu(0, \infty)<\infty$ and $c<$ $K \int_{0}^{\infty} z \nu(\mathrm{d} z)+S q$, then the take-the-money-and-run strategy is an optimal strategy for the control problem.

(iii) If $\psi^{\prime}(0+)=-\infty$, then the take-the-money-and-run strategy is an optimal strategy for the control problem.

Note that $\psi^{\prime}(0+)>-\infty$ is equivalent to $\int_{1}^{\infty} z \nu(\mathrm{d} z)<\infty$, which means that the large claims, modelled by the compound Poisson part of the process, have a finite mean. Part (iii) agrees with the intuition that if those large claims do not have a finite mean, i.e., if $\psi^{\prime}(0+)=-\infty$, then there is no economic interest in running the company/portfolio. Similarly, when $\psi^{\prime}(0+)>$ $-\infty$, one could interpret the relationship between $c$ and $K \int_{0}^{\infty} z \nu(\mathrm{d} z)+S q$ as another measure of viability. Finally, note that, when $X$ has paths of bounded variation, $c$ could be seen as the premium intensity of $X$ and that 
it is a (strictly) positive constant since we assumed that the paths of $X$ are not monotone decreasing.

Let, for $q \geq 0, W^{(q)}:(0, \infty) \rightarrow(0, \infty)$ be the scale function of the spectrally negative Lévy process $X$, which is defined as the unique continuous function with Laplace transform $\int_{0}^{\infty} \mathrm{e}^{-\theta z} W^{(q)}(z) \mathrm{d} z=1 /(\psi(\theta)-q)$ for $\theta$ large enough. More details on these functions will be given in the next section. In order to prove Theorem 1.1, we need the following monotonicity property of the scale function.

Theorem 1.2. Suppose the tail of the Lévy measure is log-convex. Then, for all $q \geq 0, W^{(q)}$ has a log-convex first derivative.

The need for a certain monotonicity property of the scale function has cropped up in several related optimal dividend problems for spectrally negative Lévy processes in order to show that a certain simple strategy is optimal. In Theorem 2 of [24] it was shown that for the original de Finetti's dividend problem (when $K=S=0$ ), a barrier strategy is optimal when $W^{(q) \prime}$ is increasing after the last point where it attains its minimum (modulo some smoothness conditions). In Theorem 3 of the same paper, it was shown that this condition on the scale function is satisfied when the Lévy measure has a completely monotone density, i.e., infinitely differentiable with derivatives alternating in sign. Then, in Theorem 2.6 of [23], this result was pushed further by assuming the weaker condition that the Lévy measure has a density which is log-convex. While dealing with the case $K=0$ and $S \in \mathbb{R}$, Loeffen [25] needed (strict) log-convexity of $W^{(q) \prime}$ in order to show that a barrier strategy or the take-the-money-and-run strategy is optimal and proved that $W^{(q) \prime}$ satisfies this if the density of the Lévy measure is completely monotone. Loeffen [26] also studied the version of the original de Finetti's problem where transaction costs are introduced and showed that when the Lévy measure has a log-convex density, then a so-called $\left(c_{1} ; c_{2}\right)$ policy is optimal; for the proof he used that under this condition on the Lévy measure, there exists numbers $0 \leq a^{\prime} \leq a^{*}$ such that $W^{(q) \prime}$ is strictly decreasing on $\left(0, a^{\prime}\right)$, constant on $\left(a^{\prime}, a^{*}\right)$ and strictly increasing on $\left(a^{*}, \infty\right)$. We also mention here the paper of Alvarez and Rakkolainen [2] in which optimal dividend problems 'of de Finetti type' are studied where the driving process is a spectrally negative Lévy diffusion with a jump component of geometric form. An important role in their analysis (cf. Theorem 3.5 of [2]) is played by a monotonicity property of a certain function which can be seen as the analogue of the scale function for spectrally negative Lévy processes.

Now, Theorem 1.2 implies that the results on optimal dividend strategies derived in [23, 24, 25, 26] are still valid when the corresponding condition imposed on the Lévy measure in these papers is replaced by the condition that the tail of the Lévy measure is log-convex. In fact, this even improves these results since the Lévy measure having a log-convex (or completely monotone) density implies that the tail of the Lévy measure is log-convex (cf. Remark 4 in [3]). There are examples for which the tail of the Lévy 
measure is log-convex, but its density is not; consider the following example taken from Section 3 of [23]:

$$
\nu(x, \infty)= \begin{cases}\mathrm{e}^{2-x} & 0<x<\frac{1}{1-\lambda}, \\ \mathrm{e}^{1-\lambda x} & x \geq \frac{1}{1-\lambda},\end{cases}
$$

where $0<\lambda<1$.

Theorem 1.1 states that the results obtained in the case $K=0$ carry over to the case when $0<K \leq 1$. Note that the case $K=0$ in Theorem 1.1 is excluded since this is a degenerate case where the condition $\psi^{\prime}(0+)>-\infty$ is irrelevant, cf. Theorem 1 of [25]. Recall that the case $S=0$ and $K=1$ was discussed in [15, 19] and that a numerical study of the behaviour of the optimal barrier level was carried out in [17]. In these three papers, the optimality of the barrier level within the family of barrier strategies is considered, not the optimality of the barrier strategy among all admissible strategies. It is important to note that a barrier strategy (or the takethe-money-and-run strategy) cannot be optimal for every jump distribution: Azcue and Muler [8] have provided a counter-example for the case $K=S=0$ and similar counter-examples can be found in other cases. This is in contrast with the dividend problem with capital injections considered in [7, 21]: there a barrier strategy always forms the optimal strategy, no matter the form of the jump measure.

The rest of the paper is organized as follows. In the next section, we state some facts about scale functions and prove Theorem 1.2. In Section 3, we compute the value function of a barrier strategy and, in Section 4 , it is shown how to determine the optimal barrier level. Finally, in Section 5, a verification lemma and Theorem 1.1 are proved. Some proofs are left for the Appendix.

\section{SCALE FUnCtions}

We first introduce some terminology. The terms positive, negative, increasing and decreasing are meant in the weak sense and for a function $f:(0, \infty) \rightarrow \mathbb{R}$ and for $0 \leq a<b \leq \infty$, we call $f$ increasing-decreasing on $(a, b)$ if there exists $c \in[a, b]$ such that $f$ is increasing on $(a, c)$ and decreasing on $(c, b)$. We call $f$ ultimately increasing if there exists $R>0$ such that $f$ is increasing on $(R, \infty)$. Finally, as $f(x)$ is defined for $x>0$, we set $f(0+):=\lim _{x \downarrow 0} f(x)$ (in case this limit is well defined). Further, for a rightcontinuous increasing function $F:[0, \infty) \rightarrow[0, \infty)$, we denote by $F(\mathrm{~d} x)$ the (unique) Borel measure on $[0, \infty)$ such that $F[0, x]=F(x)$. Conversely, for a Borel measure $F(\mathrm{~d} x)$ on $[0, \infty)$ with $F[0, x]<\infty$ for all $x \geq 0$, we will write $F(x)=F[0, x]$.

For an arbitrary spectrally negative Lévy process, the Laplace exponent $\psi$ is strictly convex and $\lim _{\theta \rightarrow \infty} \psi(\theta)=\infty$. Thus, there exists a function 
$\Phi:[0, \infty) \rightarrow[0, \infty)$ defined by $\Phi(\theta)=\sup \{\xi \geq 0 \mid \psi(\xi)=\theta\}$ (its rightinverse) and such that

$$
\psi(\Phi(\theta))=\theta, \quad \theta \geq 0 .
$$

We now recall the definition of the $(q-)$ scale function $W^{(q)}$ and some properties of this function. For further details, amongst others, on its existence and origin, we refer to [22]. The scale function of the process $X$ is defined as the unique continuous function with Laplace transform

$$
\int_{0}^{\infty} \mathrm{e}^{-\theta z} W^{(q)}(z) \mathrm{d} z=\frac{1}{\psi(\theta)-q}, \quad \text { for } \theta>\Phi(q),
$$

and this function is positive and strictly increasing. Although we primarily regard $W^{(q)}$ as a function on $(0, \infty)$, if needed, we extend the scale function to the entire real line by setting $W^{(q)}(0)=W^{(q)}(0+)$ and $W^{(q)}(x)=0$ for $x<0$. Since $W^{(q)}$ is log-concave (cf. p.89 of [25]), it is differentiable except for at most countably many points. Moreover, $W^{(q)}$ is continuously differentiable if $X$ has paths of unbounded variation or if the tail of the Lévy measure is continuous. Further, from Theorem 1 in [12, $W^{(q)}$ is twice continuously differentiable on $(0, \infty)$ if $\sigma>0$. The initial values of $W^{(q)}$ and $W^{(q) \prime}$ are given by

$$
\begin{aligned}
& W^{(q)}(0+)= \begin{cases}1 /\left(\gamma+\int_{0}^{1} z \nu(\mathrm{d} z)\right) & \text { when } \sigma=0 \text { and } \int_{0}^{1} z \nu(\mathrm{d} z)<\infty, \\
0 & \text { otherwise, }\end{cases} \\
& W^{(q)^{\prime}(0+)}= \begin{cases}2 / \sigma^{2} & \text { when } \sigma>0, \\
(\nu(0, \infty)+q) / c^{2} & \text { when } \sigma=0 \text { and } \nu(0, \infty)<\infty, \\
\infty & \text { otherwise. }\end{cases}
\end{aligned}
$$

We will also use the functions $\left\{\bar{W}^{(q)} ; q \geq 0\right\}$ defined by

$$
\bar{W}^{(q)}(x)=\int_{0}^{x} W^{(q)}(z) \mathrm{d} z .
$$

Since $W^{(q)}$ is log-concave, it follows that $\bar{W}^{(q)}$ is log-concave (cf. Prékopa [29]).

Finally, note that $W^{(q)}$ appears naturally in insurance risk theory. For the underlying/uncontrolled risk process $X$, denote the time of ruin by

$$
\tau_{0}^{-}=\inf \left\{t>0: X_{t}<0\right\}
$$

with the convention $\inf \emptyset=\infty$. Then, for $x \geq 0$, it is known that

$$
\mathbb{E}_{x}\left[\mathrm{e}^{-q \tau_{0}^{-}} \mathbb{I}_{\left\{\tau_{0}^{-}<\infty\right\}}\right]=1+q \bar{W}^{(q)}(x)-\frac{q}{\Phi(q)} W^{(q)}(x),
$$

of which the probability of ruin is a special case. 
2.1. Proof of Theorem 1.2. In Theorem 2.6, Kyprianou et al. 23] showed that, for $q>0, W^{(q) \prime}$ is strictly (increasing and) convex on $\left(a^{*}, \infty\right)$ if the Lévy measure has a log-convex density. Here $a^{*} \in[0, \infty)$ is the last point where $W^{(q) \prime}$ attains its minimum. This result was later slightly strengthened in Proposition 1 of Loeffen [26]. Kyprianou et al. 23] used that potential functions of subordinators satisfy a certain linear convolution Volterra equation and deduced this monotonicity property of $W^{(q)}$ by applying the theory of Volterra equations in combination with the well-known connection between these potential functions and the scale functions of spectrally negative Lévy processes. However, since the scale function itself satisfies a renewal equation in the case where $X$ has paths of bounded variation (see equation (11) below), one can use the theory of renewal equations to obtain a stronger result, namely Theorem 1.2. In the proof of Theorem 4.3 of Yin and Wang [34], this approach via renewal equations was undertaken as part of reproving the abovementioned result of Kyprianou et al. [23] and the contours of our Theorem 1.2, and its proof, can already be found there.

Before we give the proof of Theorem 1.2 , we need some preparatory lemmas. First we recall some facts about (log-)convex functions and sequences, and about renewal equations both in continuous and in discrete time. A function $f:(0, \infty) \rightarrow \mathbb{R}$ is convex if and only if there exists an increasing function $g:(0, \infty) \rightarrow \mathbb{R}$ such that, for a fixed $a>0$,

$$
f(x)=f(a)+\int_{a}^{x} g(y) \mathrm{d} y
$$

see, e.g., p.9-10 of [31]. This function $g$ can be taken as the left-hand derivative of $f$, which we denote by $f^{\prime-}$. Moreover, $f^{\prime-}$ is then left-continuous (cf. p.7 of [31]). This implies in particular that if $f$ is log-convex, then $\frac{\mathrm{d}^{-}}{\mathrm{d} x} \log (f(x))=f^{\prime-}(x) / f(x)$ is increasing on $(0, \infty)$. Further properties of log-convex functions are that a log-convex function is convex, the sum of two log-convex functions is log-convex and the limit of a pointwise convergent sequence of log-convex functions is log-convex (cf. p.19 of [31]).

Consider now the following renewal equation:

$$
Z(x)=1+\int_{0}^{x} Z(x-y) F(\mathrm{~d} y), \quad x \geq 0,
$$

where $F$ is a measure on $[0, \infty)$ such that $F(0)=0$ and $F(x)<\infty$ for all $x>0$. It is well known (cf. Theorem 2.4 of [4]) that the function

$$
Z(x)=\sum_{m=0}^{\infty} F^{* m}(x)
$$

is the unique solution of the above renewal equation satisfying $Z(x)<\infty$ for all $x>0$. Here, for $m \geq 0, F^{* m}(x)$ is the $m$-fold convolution of $F$, defined recursively by $F^{* 0}(x)=1$ and $F^{* m}(x)=\int_{0}^{x} F^{* m-1}(x-y) F(\mathrm{~d} y)$, for $m \geq 1$. 
For a sequence of positive real numbers $\left(f_{n}\right)_{n=1}^{\infty}$, the renewal sequence $\left(u_{n}\right)_{n=0}^{\infty}$ is defined recursively (and thus uniquely) by $u_{0}=1$ and

$$
u_{n}=\sum_{k=1}^{n} u_{n-k} f_{k}, \quad n \geq 1 .
$$

Similarly as for the continuous-time case, we have

$$
u_{n}=\sum_{m=0}^{\infty} f_{n}^{* m},
$$

where the $m$-fold convolution of $\left(f_{n}\right)_{n=0}^{\infty}$, whereby we set $f_{0}=0$, is defined recursively by $f_{n}^{* 0}=\mathbb{I}_{\{n=0\}}$ and $f_{n}^{* m}=\sum_{k=0}^{n} f_{n-k}^{* m-1} f_{k}$, for $m \geq 1$.

We denote the generating function of a sequence $\left(a_{n}\right)_{n=k}^{\infty}$ by $\widehat{a}(s)$, i.e., $\widehat{a}(s):=\sum_{n=k}^{\infty} s^{n} a_{n}$ where $s>0$ is such that $\widehat{a}(s)$ is well defined. The sequence $\left(a_{n}\right)_{n=k}^{\infty}$ is called log-convex if, for all $n \geq k, a_{n} \geq 0$ and

$$
a_{n+2} a_{n} \geq\left(a_{n+1}\right)^{2} .
$$

The following lemma is taken from De Bruijn and Erdös [13. Note that De Bruijn and Erdös [13] assume that $\sum_{n=1}^{\infty} f_{n}=1$, but that they do not use this assumption in the proof.

Lemma 2.1 (Theorem 1 of [13]). If the sequence $\left(f_{n}\right)_{n=1}^{\infty}$ is log-convex, then the renewal sequence $\left(u_{n}\right)_{n=0}^{\infty}$ is log-convex.

The next lemma is a continuous-time version of Lemma 2.1 and is based on Theorem 3.2 of Hansen and Frenk 20, in which the case where $F$ is a probability measure is dealt with. For $F$ not necessarily a probability measure, the proof is virtually the same, though we provide some more details.

Lemma 2.2. Consider an absolutely continuous measure $F(\mathrm{~d} x)=f(x) \mathrm{d} x$ on $[0, \infty)$, where $f:(0, \infty) \rightarrow[0, \infty)$ is bounded, log-convex and such that $\int_{0}^{\infty} \mathrm{e}^{-\lambda x} f(x) \mathrm{d} x<\infty$, for $\lambda$ large enough. Then $Z(x)=\sum_{n=0}^{\infty} F^{* n}(x)$ is well defined and has a log-convex density, i.e., $Z(x)=1+\int_{0}^{x} z(s) \mathrm{d} s$, where $z:(0, \infty) \rightarrow[0, \infty)$ is log-convex

Proof. Note that $\int_{0}^{\infty} \mathrm{e}^{-\lambda x} f(x) \mathrm{d} x<\infty$ for $\lambda$ large enough implies that $F(x)<$ $\infty$ for all $x>0$ and thus $Z(x)$ is well defined. Define, for $j \geq 1$, the sequence $\left(f_{n}^{(j)}\right)_{n=1}^{\infty}$ by

$$
f_{n}^{(j)}=\frac{f\left(\frac{n}{j}\right)}{j} .
$$

Then, for each $j \geq 1$, the sequence $\left(f_{n}^{(j)}\right)_{n=1}^{\infty}$ is log-convex since $f$ is logconvex. Define, for $j \geq 1$, the renewal sequence $\left(u_{n}^{(j)}\right)_{n=0}^{\infty}$ by (5), using $\left(f_{n}^{(j)}\right)_{n=1}^{\infty}$. Then, by Lemma 2.1. for each $j \geq 1$, the sequence $\left(u_{n}^{(j)}\right)_{n=0}^{\infty}$ is 
log-convex. For $a \in[0, \infty)$, denote its integer part by $\lfloor a\rfloor$. For $j \geq 1$, define the function $z_{j}:[0, \infty) \rightarrow[0, \infty)$, by

$$
z_{j}(x)=j\left(u_{n}^{(j)}\right)^{n+1-j x}\left(u_{n+1}^{(j)}\right)^{j x-n}, \quad \text { where } n=\lfloor x j\rfloor .
$$

Note that $\log \left(z_{j}\right)$ is a piecewise linear function and has an increasing leftderivative due to the $\log$-convexity of $\left(u_{n}^{(j)}\right)_{n=0}^{\infty}$. Hence $\log \left(z_{j}\right)$ is convex on $[0, \infty)$. Moreover, we have, for $n \geq 1$,

$$
\begin{aligned}
z_{j}\left(\frac{n}{j}\right) & =j u_{n}^{(j)}=j \sum_{k=1}^{n} u_{n-k}^{(j)} f_{k}^{(j)}=\sum_{k=0}^{n-1} j f_{n-k}^{(j)} u_{k}^{(j)} \\
& =\int_{0}^{n-1} f\left(\frac{n-y}{j}\right) \sum_{k=0}^{\infty} u_{k}^{(j)} \delta_{k}(\mathrm{~d} y)=\int_{0}^{\frac{n-1}{j}} f\left(\frac{n}{j}-y\right) Z_{j}(\mathrm{~d} y),
\end{aligned}
$$

where $Z_{j}(\mathrm{~d} y)=\sum_{k=0}^{\infty} u_{k}^{(j)} \delta_{\frac{k}{j}}(\mathrm{~d} y)$ and $\delta_{a}$ is the Dirac delta measure at $a$. We further define the functions $z_{j}^{(1)}$ and $z_{j}^{(2)}$ by

$$
z_{j}^{(1)}(x)=z_{j}(\lfloor x j\rfloor / j)=j u_{\lfloor x j\rfloor}^{(j)}, \quad z_{j}^{(2)}(x)=z_{j}((\lfloor x j\rfloor+1) / j)=j u_{\lfloor x j\rfloor+1}^{(j)} .
$$

Note that then we have the following inequalities for all $j \geq 1$ and for all $x \geq 0$ :

$$
z_{j}^{(1)}(x) \wedge z_{j}^{(2)}(x) \leq z_{j}(x) \leq z_{j}^{(1)}(x) \vee z_{j}^{(2)}(x) .
$$

Since $\int_{0}^{\infty} \mathrm{e}^{-\lambda x} f(x) \mathrm{d} x<\infty$ for $\lambda$ large enough, we have, by the dominated convergence theorem, that $\lim _{\lambda \rightarrow \infty} \int_{0}^{\infty} \mathrm{e}^{-\lambda x} f(x) \mathrm{d} x=0$ and thus there exists $\lambda^{*}$ such that, for all $\lambda \geq \lambda^{*}, \int_{0}^{\infty} \mathrm{e}^{-\lambda x} f(x) \mathrm{d} x<1$. It follows that by definition of the Riemann integral, we have, for $\lambda>\lambda^{*}$,

$$
\int_{0}^{\infty} \mathrm{e}^{-\lambda x} f(x) \mathrm{d} x=\lim _{j \rightarrow \infty} \sum_{n=1}^{\infty} \mathrm{e}^{-\lambda n / j} f(n / j) j^{-1}=\lim _{j \rightarrow \infty} \widehat{f^{(j)}}\left(\mathrm{e}^{-\lambda / j}\right) .
$$

Also, setting $f_{0}^{(j)}=0$ for $j \geq 1$, using (6), Fubini's theorem and a well-known property regarding generating functions and convolutions, we have, for $s>0$ such that $\widehat{f^{(j)}}(s)<1$,

$$
\begin{aligned}
\widehat{u^{(j)}}(s) & \left.=\sum_{n=0}^{\infty} s^{n} \sum_{m=0}^{\infty}\left(f^{(j)}\right)_{n}^{* m}=\sum_{m=0}^{\infty} \sum_{n=0}^{\infty} s^{n}\left(f^{(j)}\right)_{n}^{* m}=\sum_{m=0}^{\infty} \widehat{\left(f^{(j)}\right.}(s)\right)^{m} \\
& =\frac{1}{1-\widehat{f^{(j)}}(s)} .
\end{aligned}
$$

We can now deduce, for $\lambda>\lambda^{*}$, that

$$
\begin{aligned}
\lim _{j \rightarrow \infty} \int_{0}^{\infty} \mathrm{e}^{-\lambda x} Z_{j}(\mathrm{~d} x) & =\lim _{j \rightarrow \infty} \sum_{k=0}^{\infty} \mathrm{e}^{-\lambda k / j} u_{k}^{(j)}=\lim _{j \rightarrow \infty} \widehat{u}^{(j)}\left(\mathrm{e}^{-\lambda / j}\right) \\
& =\frac{1}{1-\int_{0}^{\infty} \mathrm{e}^{-\lambda x} f(x) \mathrm{d} x} .
\end{aligned}
$$


Since the right-hand side of the above equation is the Laplace transform of $Z$, it follows by the continuity theorem for Laplace transforms (see e.g. Theorem 2a in Section XIII.1 of Feller [16]) that $\lim _{j \rightarrow \infty} Z_{j}(x)=Z(x)$ for all $x \geq 0$ and this implies in particular that for all $x \geq 0$

$$
\lim _{j \rightarrow \infty} \int_{0}^{x} g(y) Z_{j}(\mathrm{~d} y)=\int_{0}^{x} g(y) Z(\mathrm{~d} y)
$$

for any bounded, continuous function $g$ on $[0, x]$.

Since $\lfloor x j\rfloor / j \rightarrow x$ as $j \rightarrow \infty$ and $f$ is bounded and continuous, we have $f(\lfloor x j\rfloor / j-y) \rightarrow f(x-y)$ as $j \rightarrow \infty$ uniformly in $y \in[0, x]$, whereby we set $f(y)=f(0+)$ for $y \leq 0$. Hence, together with 10$)$, this implies that

$$
\begin{aligned}
\lim _{j \rightarrow \infty} \mid \int_{0}^{x} f & \left(\frac{\lfloor x j\rfloor}{j}-y\right) Z_{j}(\mathrm{~d} y)-\int_{0}^{x} f(x-y) Z(\mathrm{~d} y) \mid \\
\leq & \lim _{j \rightarrow \infty}\left|\int_{0}^{x}\left[f\left(\frac{\lfloor x j\rfloor}{j}-y\right)-f(x-y)\right] Z_{j}(\mathrm{~d} y)\right| \\
& \quad+\lim _{j \rightarrow \infty}\left|\int_{0}^{x} f(x-y) Z_{j}(\mathrm{~d} y)-\int_{0}^{x} f(x-y) Z(\mathrm{~d} y)\right|=0
\end{aligned}
$$

for all $x>0$. Using (8), we can now deduce that for $x>0$,

$$
\begin{aligned}
& \lim _{j \rightarrow \infty} z_{j}^{(1)}(x)=\lim _{j \rightarrow \infty} \int_{\left[0, \frac{\lfloor x j\rfloor-1}{j}\right]} f\left(\frac{\lfloor x j\rfloor}{j}-y\right) Z_{j}(\mathrm{~d} y) \\
& \quad=\lim _{j \rightarrow \infty} \int_{[0, x]} f\left(\frac{\lfloor x j\rfloor}{j}-y\right) Z_{j}(\mathrm{~d} y)-\lim _{j \rightarrow \infty} \int_{\left.\frac{\lfloor x j\rfloor-1}{j}, x\right]} f\left(\frac{\lfloor x j\rfloor}{j}-y\right) Z_{j}(\mathrm{~d} y) \\
& \quad=\int_{0}^{x} f(x-y) Z(\mathrm{~d} y)-f(0+) \lim _{j \rightarrow \infty} Z_{j}\{\lfloor x j\rfloor / j\}=\int_{0}^{x} f(x-y) Z(\mathrm{~d} y) .
\end{aligned}
$$

In order to justify the last equality, let $\epsilon>0$ and note that since $Z\{x\}=0$, there exists $\delta>0$ such that $Z(x-\delta, x]<\epsilon$. Hence we get

$$
\lim _{j \rightarrow \infty} Z_{j}\{\lfloor x j\rfloor / j\} \leq \lim _{j \rightarrow \infty} Z_{j}(x-\delta, x]=Z(x-\delta, x]<\epsilon,
$$

which shows that $\lim _{j \rightarrow \infty} Z_{j}\{\lfloor x j\rfloor / j\}=0$. Similarly, we can deduce that $\lim _{j \rightarrow \infty} z_{j}^{(2)}(x)=\int_{0}^{x} f(x-y) Z(\mathrm{~d} y)$ and thus, by (9), we have that for all $x>0$,

$$
z(x):=\lim _{j \rightarrow \infty} z_{j}(x)=\int_{0}^{x} f(x-y) Z(\mathrm{~d} y) .
$$

It follows that $z$ is log-convex, since it is the limit of a convergent sequence of $\log$-convex functions. We now finish the proof by showing that $z$ is a density 
of $Z$. Indeed, we have for $\lambda>\lambda^{*}$,

$$
\begin{aligned}
\int_{0}^{\infty} \mathrm{e}^{-\lambda x}\left(1+\int_{0}^{x} z(s) \mathrm{d} s\right) \mathrm{d} x & =\frac{1}{\lambda}+\frac{1}{\lambda} \int_{0}^{\infty} \mathrm{e}^{-\lambda x} f(x) \mathrm{d} x \int_{0}^{\infty} \mathrm{e}^{-\lambda x} Z(\mathrm{~d} x) \\
& =\frac{1}{\lambda}\left(1+\frac{\int_{0}^{\infty} \mathrm{e}^{-\lambda x} f(x) \mathrm{d} x}{1-\int_{0}^{\infty} \mathrm{e}^{-\lambda x} f(x) \mathrm{d} x}\right) \\
& =\int_{0}^{\infty} \mathrm{e}^{-\lambda x} Z(x) \mathrm{d} x
\end{aligned}
$$

which implies $Z(x)=1+\int_{0}^{x} z(s) \mathrm{d} s$.

The next lemma is the final lemma we need before we prove Theorem 1.2 . Note that part (i) is Lemma 8.1.6 of Müller and Stoyan [27.

Lemma 2.3. $\quad$ (i) Let $f:(0, \infty) \rightarrow \mathbb{R}$ be differentiable. Then $f^{\prime}$ is logconvex if and only if, for all $h>0$, the function

$$
x \mapsto f(x+h)-f(x)
$$

is log-convex.

(ii) Suppose $\left(f_{n}\right)_{n \geq 1}$ is a sequence of differentiable functions on $(0, \infty)$ which converges pointwise to another differentiable function $f$. If $f_{n}^{\prime}$ is $\log$-convex for all $n \geq 1$, then $f^{\prime}$ is log-convex.

Proof. (i) For the necessity part, fix $h>0$ and, for $\delta>0$, let $T_{\delta}=\{0=$ $\left.t_{0}^{(\delta)}, t_{1}^{(\delta)}, \ldots, t_{n(\delta)}^{(\delta)}=h\right\}$ be a finite partition of the interval $[0, h]$ such that $\max _{0 \leq i \leq n(\delta)}\left|t_{i}-t_{i-1}\right| \leq \delta$. Define the functions $g_{\delta}$ by

$$
g_{\delta}(x)=\sum_{i=1}^{n(\delta)} f^{\prime}\left(x+t_{i}^{(\delta)}\right)\left(t_{i}^{(\delta)}-t_{i-1}^{(\delta)}\right) .
$$

Since for a given $c \geq 0, x \mapsto f^{\prime}(x+c)$ is log-convex and the sum of logconvex functions is log-convex, it follows that $g_{\delta}$ is log-convex. By definition of the Riemann integral, $\lim _{\delta \downarrow 0} g_{\delta}(x)=\int_{x}^{x+h} f^{\prime}(y) \mathrm{d} y=f(x+h)-f(x)$, and since the pointwise limit of a convergent sequence of log-convex functions is log-convex, it follows that $f(x+h)-f(x)$ is log-convex.

To prove the sufficiency part, we use again that a convergent sequence of log-convex functions is log-convex together with

$$
f^{\prime}(x)=\lim _{h \downarrow 0} \frac{f(x+h)-f(x)}{h} .
$$

(ii) Since $f_{n}^{\prime}$ is log-convex, it follows by (i) that, for all $h>0, f_{n}(x+h)-$ $f_{n}(x)$ is log-convex. Fix $x, y>0$ and $\lambda \in[0,1]$. Thus,

$$
\begin{aligned}
\log \left[f_{n}(\lambda x+\right. & \left.(1-\lambda) y+h)-f_{n}(\lambda x+(1-\lambda) y)\right] \\
& \leq \lambda \log \left[f_{n}(x+h)-f_{n}(x)\right]+(1-\lambda) \log \left[f_{n}(y+h)-f_{n}(y)\right] .
\end{aligned}
$$


Taking limits as $n \rightarrow \infty$ gives that the above inequality holds with $f_{n}$ replaced by $f$ and thus for each $h>0, f(x+h)-f(x)$ is log-convex. Using (i), we conclude that $f^{\prime}$ is log-convex.

Proof of Theorem 1.2. We recall that $W^{(q)}$ is the scale function of the spectrally negative Lévy process $X$ with Lévy triplet $(\gamma, \sigma, \nu)$ and Laplace exponent $\psi$. Note that $W^{(q)}$ is differentiable since the tail of the Lévy measure is log-convex and thus continuous. We prove the theorem in three steps.

Step 1: Assume that $\nu(0, \infty)<\infty$ and $\sigma=0$. Then we can write $\psi(\lambda)=$ $c \lambda-\int_{0}^{\infty}\left(1-\mathrm{e}^{-\lambda x}\right) \nu(\mathrm{d} x)=\lambda\left(c-\int_{0}^{\infty} \mathrm{e}^{-\lambda x} \nu(x, \infty) \mathrm{d} x\right)$, where $c=\gamma+\int_{0}^{1} x \nu(\mathrm{d} x)$. The scale function $W^{(q)}$ satisfies the following renewal equation

$$
c W^{(q)}(x)=1+\int_{0}^{x} W^{(q)}(x-y)(\nu(y, \infty)+q) \mathrm{d} y,
$$

which can be proved by showing that the Laplace transforms of both sides of the equation are equal, hereby noting that both sides are continuous in $x$. Since $W^{(q)}(x)<\infty$ for all $x>0$, it follows that $W^{(q)}(x)=\frac{1}{c} \sum_{m=0}^{\infty} F^{* m}(x)$, where $F(x)=\frac{1}{c} \int_{0}^{x}(\nu(y, \infty)+q) \mathrm{d} y($ cf. (4) $)$ and so, by Lemma 2.2., $W^{(q) \prime}$ is log-convex.

Step 2: Assume that $\nu(0, \infty)=\infty$ and $\sigma=0$. We write $\bar{\nu}(x)=\nu(x, \infty)$. Since $\bar{\nu}(x)$ is log-convex, $\bar{\nu}^{-}(x)$, the left-hand derivative of $\bar{\nu}$ at $x$, is well defined for all $x>0$ and, moreover, $\nu(\mathrm{d} x)=-\bar{\nu}^{\prime-}(x) \mathrm{d} x$. Define now, for $n \geq 1$, the measure $\nu_{n}(\mathrm{~d} x)$ by

$$
\nu_{n}(x, \infty)= \begin{cases}\bar{\nu}(1 / n) \exp \left\{\frac{\bar{\nu}^{\prime}-(1 / n)}{\bar{\nu}(1 / n)}\left(x-\frac{1}{n}\right)\right\} & 0<x<1 / n, \\ \bar{\nu}(x) & x \geq 1 / n .\end{cases}
$$

Then, for each $n \geq 1, \nu_{n}(0, \infty)<\infty$ and $\bar{\nu}_{n}(x):=\nu_{n}(x, \infty)$ is log-convex. Let $W_{n}^{(q)}$ be the scale function corresponding to the Lévy triplet $\left(\gamma, 0, \nu_{n}\right)$ and denote the corresponding Laplace exponent by $\psi_{n}$. From the first step, we know that $W_{n}^{(q) \prime}$ is log-convex.

Further, by the log-convexity of $\bar{\nu}$, we have that $\frac{\bar{\nu}^{\prime}(x)}{\bar{\nu}(x)}$ is an increasing (and negative) function and thus for $0<x \leq 1 / n$,

$$
\frac{\mathrm{d}^{-}}{\mathrm{d} x} \log \left(\bar{\nu}_{n}(x)\right)=\frac{\bar{\nu}_{n}^{\prime-}(x)}{\bar{\nu}_{n}(x)}=\frac{\bar{\nu}^{\prime-}(1 / n)}{\bar{\nu}(1 / n)} \geq \frac{\bar{\nu}^{\prime-}(x)}{\bar{\nu}(x)}=\frac{\mathrm{d}^{-}}{\mathrm{d} x} \log (\bar{\nu}(x)) .
$$

Since $\log \left(\bar{\nu}_{n}(1 / n)\right)=\log (\bar{\nu}(1 / n))$, inequality 12 implies that $\log \left(\bar{\nu}_{n}(x)\right) \leq$ $\log (\bar{\nu}(x))$ and thus $\bar{\nu}_{n}(x) \leq \bar{\nu}(x)$ for all $0<x \leq 1 / n$. Now using (12) again, we conclude that

$$
\bar{\nu}^{\prime-}(x) \leq \bar{\nu}_{n}^{\prime-}(x) \leq 0, \quad \text { for all } 0<x \leq 1 / n .
$$


We can now use the dominated convergence theorem to deduce that for all $\theta \geq 0$

$$
\begin{aligned}
\lim _{n \rightarrow \infty} \psi_{n}(\theta) & =\gamma \theta+\lim _{n \rightarrow \infty} \int_{0}^{\infty}\left(1-\mathrm{e}^{-\theta x}-\theta x \mathbb{I}_{(0,1]}(x)\right) \bar{\nu}_{n}^{\prime-}(x) \mathrm{d} x \\
& =\gamma \theta+\int_{0}^{\infty}\left(1-\mathrm{e}^{-\theta x}-\theta x \mathbb{I}_{(0,1]}(x)\right) \bar{\nu}^{\prime-}(x) \mathrm{d} x \\
& =\psi(\theta)
\end{aligned}
$$

and this implies that the Laplace transform of $W_{n}^{(q)}$ converges as $n \rightarrow \infty$ to the Laplace transform of $W^{(q)}$. Hence, by the continuity theorem for Laplace transforms, we have $\lim _{n \rightarrow \infty} W_{n}^{(q)}(x)=W^{(q)}(x)$ for all $x \geq 0$ and so we conclude by Lemma 2.3 (ii) that $W^{(q) \prime}$ is log-convex.

Step 3: Assume that $\sigma>0$. Define for $n \geq 1$, the measure $\nu_{n}(\mathrm{~d} x)$ by

$$
\nu_{n}(x, \infty)=\nu(x, \infty)+\frac{1}{2} \sigma^{2} n^{2} \mathrm{e}^{-n x} .
$$

Then, for each $n \geq 1, \nu_{n}$ is a Lévy measure and $\nu_{n}(x, \infty)$ is log-convex on $(0, \infty)$. Define $\gamma_{n}=\gamma+\frac{1}{2} \sigma^{2} n \mathrm{e}^{-n}(n+1)$, let $W_{n}^{(q)}$ be the scale function corresponding to the Lévy triplet $\left(\gamma_{n}, 0, \nu_{n}\right)$, and denote the corresponding Laplace exponent by $\psi_{n}$. From the second step, we know that $W_{n}^{(q) \prime}$ is logconvex. Further, for all $\theta \geq 0$,

$$
\begin{aligned}
\lim _{n \rightarrow \infty} \psi_{n}(\theta) & =\psi(\theta)-\frac{1}{2} \sigma^{2} \theta^{2}+\lim _{n \rightarrow \infty}\left\{\frac{1}{2} \sigma^{2} n \mathrm{e}^{-n}(n+1) \theta\right. \\
& \left.-\int_{0}^{\infty}\left(1-\mathrm{e}^{-\theta x}-\theta x \mathbb{I}_{(0,1]}(x)\right) \frac{1}{2} \sigma^{2} n^{3} \mathrm{e}^{-n x} \mathrm{~d} x\right\} \\
& =\psi(\theta)-\frac{1}{2} \sigma^{2} \theta^{2}+\frac{1}{2} \sigma^{2} \lim _{n \rightarrow \infty}\left\{\frac{n^{3}}{\theta+n}-n^{2}+\theta n\right\} \\
& =\psi(\theta)
\end{aligned}
$$

and thus, by the continuity theorem for Laplace transforms, $W_{n}^{(q)}(x) \rightarrow$ $W^{(q)}(x)$ as $n \rightarrow \infty$ for all $x \geq 0$. Now, by Lemma 2.3(ii), we deduce that $W^{(q) \prime}$ is log-convex.

\section{VALUe FUnCtion of A BARRIER STRATEGY}

For the rest of the paper we make the assumption that the scale function is continuously differentiable on $(0, \infty)$. The proof of the lemma below is given in the Appendix.

Lemma 3.1. Suppose $\psi^{\prime}(0+)>-\infty$. Then, for all $q>0$ and $x \geq 0$,

$$
\mathbb{E}_{x}\left[\mathrm{e}^{-q \tau_{0}^{-}} X_{\tau_{0}^{-}} \mathbb{I}_{\left\{\tau_{0}^{-}<\infty\right\}}\right]>-\infty
$$


and, for $0 \leq x \leq b$,

$$
\mathbb{E}_{x}\left[\mathrm{e}^{-q \sigma^{b}} U_{\sigma^{b}}^{b}\right]>-\infty .
$$

We are now ready to compute the value function of a horizontal barrier strategy.

Lemma 3.2. Assume $\psi^{\prime}(0+)>-\infty$. For $b \geq 0$, the value function of $a$ barrier strategy at level $b$ is given by

$$
v_{b}(x)= \begin{cases}S+\int_{0}^{x} A(y) \mathrm{d} y+\frac{1-A(b)}{W^{(q) \prime}(b)} W^{(q)}(x), & \text { if } 0 \leq x \leq b, \\ x-b+v_{b}(b), & \text { if } x>b,\end{cases}
$$

where

$$
A(y)=K\left(1-\psi^{\prime}(0+) W^{(q)}(y)+q \bar{W}^{(q)}(y)\right)+S q W^{(q)}(y) .
$$

Proof. The following was established in [6] (Theorem 1 and Remark 4): for $q, r \geq 0$, we have

$$
\begin{aligned}
\mathbb{E}_{x}\left[\mathrm{e}^{\left.-q \sigma^{b}+r U_{\sigma^{b}}^{b}\right]=}\right. & \mathrm{e}^{r x}+(q-\psi(r)) \int_{0}^{x} \mathrm{e}^{r(x-z)} W^{(q)}(z) \mathrm{d} z \\
& -W^{(q)}(x) \frac{r \mathrm{e}^{r b}+(q-\psi(r)) \int_{0}^{b} \mathrm{e}^{r(b-z)} W^{(q)}(\mathrm{d} z)}{W^{(q) \prime}(b)} .
\end{aligned}
$$

Taking the right-hand derivative with respect to $r$ on both sides and then evaluating at zero leads to, with the help of the dominated convergence theorem and (14), which allows switching expectation and derivative,

$$
\begin{aligned}
\mathbb{E}_{x}\left[\mathrm{e}^{-q \sigma^{b}} U_{\sigma^{b}}^{b}\right]=x-\psi^{\prime}(0+) \bar{W}^{(q)}(x) & +q \int_{0}^{x} \bar{W}^{(q)}(y) \mathrm{d} y \\
& -W^{(q)}(x) \frac{1-\psi^{\prime}(0+) W^{(q)}(b)+q \bar{W}^{(q)}(b)}{W^{(q)^{\prime}}(b)} .
\end{aligned}
$$

The result follows by combining the above with Proposition 2 in [25].

\section{Determining the optimal barrier level}

For $x \geq 0$, define $F(x)=\frac{1-A(x)}{W^{(q) \prime}(x)}$, with $A$ given by $(15)$, and then define

$$
b^{*}=\sup \{b \geq 0: F(b) \geq F(x), \forall x \geq 0\},
$$

the candidate for the optimal barrier level. We mention here that it is well known that, as $q>0, \lim _{x \rightarrow \infty} W^{(q)^{\prime}}(x)=\infty$ and therefore by the logconcavity of $W^{(q)}$, we must have that $W^{(q) \prime}$ is strictly positive and thus $F$ is well defined and continuous on $(0, \infty)$. The proof of the following lemma is provided in the Appendix.

Lemma 4.1. Assume $\psi^{\prime}(0+)>-\infty$. The function $F$ cannot be ultimately increasing. This implies, in particular, that $b^{*}<\infty$.

The following is a key step in proving part (i) of Theorem 1.1. 
Lemma 4.2. Assume $\psi^{\prime}(0+)>-\infty$. If $W^{(q) \prime}$ is log-convex on $(0, \infty)$, then $F$ is increasing-decreasing on $(0, \infty)$. In particular, $F$ is decreasing on $\left(b^{*}, \infty\right)$.

Proof. Recall that since $W^{(q) \prime}$ is log-convex and thus convex on $(0, \infty)$, one can write for some fixed $a>0$,

$$
W^{(q) \prime}(x)=W^{(q) \prime}(a)+\int_{a}^{x} W^{(q) \prime \prime}-(z) \mathrm{d} z,
$$

where $W^{(q) \prime \prime-}$ is the left-hand derivative of $W^{(q) \prime}$. Recall also that $W^{(q) \prime \prime-}$ is a left-continuous and increasing function. Since $W^{(q) \prime \prime-}$ is increasing and $\lim _{x \rightarrow \infty} W^{(q)^{\prime}}(x)=\infty$, we have, for some $0 \leq a^{\prime} \leq a^{*}<\infty$, that

$$
W^{(q) \prime \prime}(x) \begin{cases}<0 & \text { for all } x \in\left(0, a^{\prime}\right) \\ =0 & \text { for all } x \in\left(a^{\prime}, a^{*}\right) \\ >0 & \text { for all } x \in\left(a^{*}, \infty\right)\end{cases}
$$

which correspond to $W^{(q) \prime}$ being strictly decreasing, constant and strictly increasing, respectively.

We now introduce the following functions related to $F$. For $x>0$, define

$$
\begin{gathered}
f(x)=\frac{1-A(x)}{W^{(q)}(x)}, \quad g(x)=\frac{-A^{\prime}(x)}{W^{(q) \prime}(x)}, \\
G(x)=\frac{-A^{\prime}(x)}{W^{(q) \prime \prime}-(x)}, x \in\left(0, a^{\prime}\right) \cup\left(a^{*}, \infty\right) .
\end{gathered}
$$

It is easily seen that the following hold:

$$
\begin{gathered}
F(x)=\frac{W^{(q)}(x)}{W^{(q) \prime}(x)} f(x), \quad G(x)=\frac{W^{(q) \prime}(x)}{W^{(q) \prime \prime}-(x)} g(x), x \in\left(0, a^{\prime}\right) \cup\left(a^{*}, \infty\right), \\
F^{\prime-}(x)=\frac{W^{(q) \prime \prime}-(x)}{W^{(q) \prime}(x)}(G(x)-F(x)), x \in\left(0, a^{\prime}\right) \cup\left(a^{*}, \infty\right), \\
f^{\prime}(x)=\frac{W^{(q) \prime}(x)}{W^{(q)}(x)}(g(x)-f(x)),
\end{gathered}
$$

and

$$
F^{\prime}(x)=g(x), x \in\left(a^{\prime}, a^{*}\right) .
$$

Note that since $F$ is the ratio of two absolutely continuous functions where the corresponding denominator is nowhere zero, $F$ itself is absolutely continuous. Hence for $0<x_{1}<x_{2}, F\left(x_{2}\right)=F\left(x_{1}\right)+\int_{x_{1}}^{x_{2}} F^{\prime-}(y) \mathrm{d} y$ and so the sign of $F^{\prime-}$ on $\left(x_{1}, x_{2}\right)$ determines precisely where $F$ is increasing or decreasing on $\left(x_{1}, x_{2}\right)$.

Since

$$
g(x)=K \psi^{\prime}(0+)-S q-K q \frac{W^{(q)}(x)}{W^{(q) \prime}(x)},
$$


$K>0$ and $W^{(q)}$ is log-concave, it follows that $g$ is a decreasing function. Together with the log-convexity of $W^{(q) \prime}$, 17) and (18), we get

(22) if $g(x) \leq 0$ for all $x \in(c, d) \subseteq\left(0, a^{\prime}\right)$, then $G$ is increasing on $(c, d)$,

(23) if $g(x) \geq 0$ for all $x \in(c, d) \subseteq\left(a^{*}, \infty\right)$, then $G$ is decreasing on $(c, d)$.

Further, using (17) and (19), we immediately see that, for $x \in\left(0, a^{\prime}\right)$,

$$
\begin{array}{lll}
F^{\prime-}(x) \geq 0 & \text { if and only if } & F(x) \geq G(x), \\
F^{\prime-}(x) \leq 0 & \text { if and only if } & F(x) \leq G(x),
\end{array}
$$

and, for $x \in\left(a^{*}, \infty\right)$,

$$
\begin{array}{lll}
F^{\prime-}(x) \geq 0 & \text { if and only if } & F(x) \leq G(x), \\
F^{\prime-}(x) \leq 0 & \text { if and only if } & F(x) \geq G(x),
\end{array}
$$

Moreover, with a little bit more work, the following are established:

$$
\begin{aligned}
& \text { if } x \in\left(0, a^{\prime}\right) \text { and } g(x) \geq 0, \text { then } F^{\prime-}(x) \geq 0, \\
& \text { if } x \in\left(a^{*}, \infty\right) \text { and } g(x) \leq 0 \text {, then } F^{\prime-}(x) \leq 0 .
\end{aligned}
$$

To see this, first note that

$$
f(x)=K \psi^{\prime}(0+)-S q+\frac{1-K}{W^{(q)}(x)}-K q \frac{\bar{W}^{(q)}(x)}{W^{(q)}(x)},
$$

and since $0<K \leq 1$ and $\bar{W}$ is log-concave, it follows that $f$ is decreasing on $(0, \infty)$. Hence by $(20), f(x) \geq g(x)$, for all $x>0$. Secondly, note that by the log-concavity of $W^{(q)}$ and $(17)$ we have

$$
\left[\frac{W^{(q)}(x)}{W^{(q) \prime}(x)}-\frac{W^{(q) \prime}(x)}{W^{(q) \prime \prime}-(x)}\right] \begin{cases}\geq 0 & \text { on }\left(0, a^{\prime}\right), \\ \leq 0 & \text { on }\left(a^{*}, \infty\right) .\end{cases}
$$

Finally, if the conditions in (28), respectively in (29), hold, then, using that $f(x) \geq g(x)$, we have

$$
F(x)-G(x) \geq g(x)\left[\frac{W^{(q)}(x)}{W^{(q) \prime}(x)}-\frac{W^{(q) \prime}(x)}{W^{(q)^{\prime \prime}-}(x)}\right] \geq 0,
$$

and, using (24), respectively (27), we deduce the conclusions in (28), respectively 290 .

With the above relations established, we now proceed with proving that $F$ is increasing-decreasing. Define $r_{g}=\inf \{x \geq 0: g(x)<0\}$, using again the convention $\inf \emptyset=\infty$. We split the analysis into three cases:

- Assume $r_{g}<a^{\prime}$. Then (28) implies that $F$ is increasing on $\left(0, r_{g}\right)$. Now let $p=\inf \left\{x \in\left(r_{g}, a^{\prime}\right): F(x)<G(x)\right\}$, whereby we set $p=a^{\prime}$ if $F \geq G$ on $\left(r_{g}, a^{\prime}\right)$. Then by (24), $F$ is increasing on $\left(r_{g}, p\right)$ and by (25) and the fact that $G$ is increasing on $\left(r_{g}, a^{\prime}\right)$ because of $(22)$, we have that $F$ is decreasing on $\left(p, a^{\prime}\right)$. Finally, due to (21) and (29), $F$ is decreasing on $\left(a^{\prime}, \infty\right)$.

- Assume $a^{\prime} \leq r_{g} \leq a^{*}$. Then (28) implies that $F$ is increasing on $\left(0, a^{\prime}\right)$, while 21) implies that $F$ is increasing on $\left(a^{\prime}, r_{g}\right)$ and decreasing on $\left(r_{g}, a^{*}\right)$, 
and finally (29) implies that $F$ is decreasing on $\left(a^{*}, \infty\right)$. So, in this case, $b^{*}=r_{g}$.

- Assume $a^{*}<r_{g} \leq \infty$. Then (28) implies that $F$ is increasing on $\left(0, a^{\prime}\right)$ while (21) implies that $F$ is increasing on $\left(a^{\prime}, a^{*}\right)$. Before going any further we prove the following:

$$
\text { suppose } F(p)=G(p) \text { for some } p \in\left(a^{*}, r_{g}\right) \text {, then } F \geq G \text { on }\left(p, r_{g}\right) \text {. }
$$

Fix $x \in\left(p, r_{g}\right)$ and let $\bar{z}=\sup \{z \in(p, x): F(z) \geq G(z)\}$. By continuity of $F$ and left-continuity of $G, F(\bar{z}) \geq G(\bar{z})$. If $\bar{z}=x$, then $F(x) \geq G(x)$. If $\bar{z}<x$, then $F(z)<G(z)$ for all $z \in(\bar{z}, x)$, which implies $F^{\prime-}(z) \geq 0$ for all $z \in(\bar{z}, x)$ by (26) and thus $F(x)=F(\bar{z})+\int_{\bar{z}}^{x} F^{\prime-}(z) \mathrm{d} z \geq F(\bar{z}) \geq G(\bar{z})$. Since $G$ is decreasing on $\left(a^{*}, r_{g}\right)$ by $(23)$, we have $G(\bar{z}) \geq G(x)$ and thus $F(x) \geq G(x)$. This proves $(30)$.

Now let $p=\inf \left\{x \in\left(a^{*}, r_{g}\right): F(x) \geq G(x)\right\}$, whereby we set $p=r_{g}$ if $F<G$ on $\left(a^{*}, r_{g}\right)$. Then by (26), $F$ is increasing on $\left(a^{*}, p\right)$ and by (30) and (27), $F$ is decreasing on $\left(p, r_{g}\right)$. Note that this forces that $p<\infty$ since $F$ cannot be ultimately increasing (cf. Lemma 4.1). Finally, due to 29$), F$ is decreasing on $\left(r_{g}, \infty\right)$.

\section{VERIFICATION LEMMA}

Lemma 5.1. Suppose $\pi$ is an admissible dividend strategy such that $v_{\pi}$ is continuously differentiable on $(0, \infty)$ and $v_{\pi}^{\prime}$ is absolutely continuous on $(0, \infty)$ with a density which is bounded on sets of the form $[1 / n, n], n \geq 1$. Suppose further that for all $x>0, v_{\pi}^{\prime}(x) \geq 1$ and $(\Gamma-q) v_{\pi}(x) \leq 0$, where $\Gamma$ is the operator defined by

$$
\Gamma v(x)=\gamma v^{\prime}(x)+\frac{\sigma^{2}}{2} v^{\prime \prime}(x)+\int_{0}^{\infty}\left(v(x-z)-v(x)+v^{\prime}(x) z \mathbb{I}_{(0,1]}(z)\right) \nu(\mathrm{d} z) .
$$

Here $v$ is a function on $\mathbb{R}$ such that $\Gamma v(x)$ is well defined for all $x>0$. Suppose further that $v_{\pi}(0) \geq S$. Then $\pi$ is an optimal strategy for the control problem.

Proof. Noting that $v_{\pi}(x) \geq S$ for $x \geq 0$ by the assumptions and $v_{\pi}(x)=$ $S+K x$ for $x<0$ by definition, we see that the proof is virtually identical to the proof of Lemma 1 in [25. Hereby we note that since $v_{\pi}$ is not twice continuously differentiable, instead of the standard Itô's formula (cf. Theorem II.32 of [30]), one has to appeal to the Meyer-Itô formula (cf. Theorem IV.71 of [30]).

Before we prove the main result, we need one more lemma. Again the proof is given in the Appendix.

Lemma 5.2. Assume $\psi^{\prime}(0+)>-\infty$ and $W^{(q) \prime}$ is absolutely continuous on $(0, \infty)$ with a density which is bounded on sets of the form $[1 / n, n], n \geq 1$. Then for all $x>0,(\Gamma-q) W^{(q)}(x)=0$ and $(\Gamma-q)\left(S+\int_{0}^{x} A(y) \mathrm{d} y\right)=0$. 
Proof of Theorem 1.1. In each case, we will show that $v_{b^{*}}$, or $v_{\text {run }}$, satisfies the conditions of Lemma 5.1. Note that by Theorem 1.2, $W^{(q) \prime}$ is log-convex.

(i). Since $W^{(q) \prime}$ is log-convex and $\lim _{x \uparrow b^{*}} v_{b^{*}}^{\prime}(x)=1$, it can be easily verified that $v_{b^{*}}$ satisfies the required smoothness conditions of Lemma 5.1. By the definition of $b^{*}$, we have for all $x \leq b^{*}$,

$$
v_{b^{*}}^{\prime}(x)=A(x)+F\left(b^{*}\right) W^{(q)^{\prime}}(x) \geq A(x)+F(x) W^{(q) \prime}(x)=1 .
$$

Further, using Lemma 5.2, we clearly have that $(\Gamma-q) v_{b^{*}}(x)=0$ for all $0<x<b^{*}$ and by continuity we have $(\Gamma-q) v_{b^{*}}\left(b^{*}\right)=0$ (cf. Lemma 4.1 of [23]). On the other hand, for all $x>b^{*}$, we have

$$
\begin{aligned}
&(\Gamma-q) v_{b^{*}}(x) \\
&=(\Gamma-q) v_{b^{*}}(x)-(\Gamma-q)\left(S+\int_{0}^{x} A(y) \mathrm{d} y\right)-F(x)(\Gamma-q) W^{(q)}(x) \\
&=-\frac{\sigma^{2}}{2}\left(A^{\prime}(x)+F(x) W^{(q) \prime \prime}-(x)\right)-q\left(v_{b^{*}}-v_{x}\right)(x) \\
& \quad+\int_{0}^{\infty}\left(\left(v_{b^{*}}-v_{x}\right)(x-z)-\left(v_{b^{*}}-v_{x}\right)(x)\right) \nu(\mathrm{d} z) .
\end{aligned}
$$

If $\sigma>0$, then $W^{(q)}$ is twice continuously differentiable which implies that $F$ is continuously differentiable and thus by Lemma 4.2 . $A^{\prime}(x)+F(x) W^{(q) \prime \prime}(x)=$ $-W^{(q) \prime}(x) F^{\prime}(x) \geq 0$ for $x>b^{*}$. For $u \in\left[0, b^{*}\right],\left(v_{b^{*}}^{\prime}-v_{x}^{\prime}\right)(u)=\left(F\left(b^{*}\right)-\right.$ $F(x)) W^{(q) \prime}(u) \geq 0$ by definition of $b^{*}$ and, for $u \in\left(b^{*}, x\right]$,

$\left(v_{b^{*}}^{\prime}-v_{x}^{\prime}\right)(u)=1-\left[A(u)+F(x) W^{(q) \prime}(u)\right] \geq 1-\left[A(u)+F(u) W^{(q) \prime}(u)\right]=0$,

by Lemma 4.2. This implies that $\left(v_{b^{*}}-v_{x}\right)(u)-\left(v_{b^{*}}-v_{x}\right)(x) \leq 0$ for all $u \in[0, x]$ and that $\left(v_{b^{*}}-v_{x}\right)(x) \geq 0$, since $\left(v_{b^{*}}-v_{x}\right)(0+)=\left(F\left(b^{*}\right)-\right.$ $F(x)) W^{(q)}(0+) \geq 0$ by definition of $b^{*}$. Note also that $\left(v_{b^{*}}-v_{x}\right)(u)=0$ for $u<0$. Putting all this together gives us $(\Gamma-q) v_{b^{*}}(x) \leq 0$ for $x>b^{*}$.

In order to show the last condition of Lemma 5.1, note that $v_{b^{*}}(0)=$ $S+F\left(b^{*}\right) W^{(q)}(0+)$ and so it is enough if we show that $F(0+) \geq 0$. However, this follows from the assumptions on the Lévy triplet and the values of $W^{(q)}(0+)$ and $W^{(q) \prime}(0+)$ given in (2).

(ii). Since $v_{\text {run }}(x)=x+S$ for $x \geq 0$, the only non-trivial thing to show is that $(\Gamma-q) v_{\text {run }}(x) \leq 0$ for all $x>0$. Since $\nu(0, \infty)<\infty$ and $\int_{x}^{\infty} z \nu(\mathrm{d} z)<\infty$ 
for all $x>0$ by assumption, one can write

$$
\begin{aligned}
(\Gamma & -q) v_{\text {run }}(x) \\
& =c-\int_{0}^{x} z \nu(\mathrm{d} z)+K \int_{x}^{\infty}(x-z) \nu(\mathrm{d} z)-x \nu(x, \infty)-q(x+S) \\
& =c-\int_{0}^{\infty} z \nu(\mathrm{d} z)+(1-K) \int_{x}^{\infty}(z-x) \nu(\mathrm{d} z)-q(x+S) \\
& =c-K \int_{0}^{\infty} z \nu(\mathrm{d} z)-S q+(1-K) \int_{0}^{\infty} z(\nu(x+\mathrm{d} z)-\nu(\mathrm{d} z))-q x .
\end{aligned}
$$

Because the tail of $\nu$ is log-convex, $\nu$ has in particular a decreasing density. Together with the other assumptions on the Lévy triplet and the fact that $K \leq 1$, we get that $(\Gamma-q) v_{\text {run }}(x) \leq 0$ for all $x>0$.

(iii). Again the only non-trivial thing to show is that $(\Gamma-q) v_{\text {run }}(x) \leq 0$ for all $x>0$. We have

$$
\begin{aligned}
& (\Gamma-q) v_{\text {run }}(x)= \\
& \begin{cases}\gamma-\int_{1}^{x} z \nu(\mathrm{d} z)+(K-1) x \nu(x, \infty)-K \int_{x}^{\infty} z \nu(\mathrm{d} z)-q(x+S), & x \geq 1, \\
\gamma+\int_{x}^{1} z \nu(\mathrm{d} z)+(K-1) x \nu(x, \infty)-K \int_{x}^{\infty} z \nu(\mathrm{d} z)-q(x+S), & x<1 .\end{cases}
\end{aligned}
$$

Since in this case $\int_{x}^{\infty} z \nu(\mathrm{d} z)=\infty$ for all $x>0$, and since $K>0$, it follows that $(\Gamma-q) v_{\text {run }}(x)=-\infty$ for all $x>0$.

\section{ACKnowledgements}

The second author is supported by a grant from the Natural Sciences and Engineering Research Council of Canada.

\section{Appendix}

Proof of Lemma 3.1. Using a standard computation involving the compensation formula (see e.g. Section 8.4 in [22]), we obtain an expression for

the Gerber-Shiu function consisting of the first moment of the deficit at ruin:

$$
\begin{aligned}
\mathbb{E}_{x}\left[\mathrm{e}^{-q \tau_{0}^{-}} X_{\tau_{0}^{-}}\right. & \left.\mathbb{I}_{\left\{\tau_{0}^{-}<\infty\right\}}\right] \\
& =\int_{0}^{\infty} \int_{y}^{\infty}(y-\theta) \nu(\mathrm{d} \theta) \int_{0}^{\infty} \mathrm{e}^{-q t} \mathbb{P}_{x}\left(X_{t} \in \mathrm{d} y, t<\tau_{0}^{-}\right) \mathrm{d} t,
\end{aligned}
$$

where

$$
\int_{0}^{\infty} \mathrm{e}^{-q t} \mathbb{P}_{x}\left(X_{t} \in \mathrm{d} y, t<\tau_{0}^{-}\right) \mathrm{d} t=\left\{\mathrm{e}^{-\Phi(q) y} W^{(q)}(x)-W^{(q)}(x-y)\right\} \mathrm{d} y
$$


(see Corollary 8.8 in [22]). We deduce

$$
\begin{aligned}
\left|\int_{1}^{\infty} \int_{y}^{\infty}(y-\theta) \nu(\mathrm{d} \theta)\left\{\mathrm{e}^{-\Phi(q) y} W^{(q)}(x)-W^{(q)}(x-y)\right\} \mathrm{d} y\right| \\
\quad \leq \int_{1}^{\infty} \theta \nu(\mathrm{d} \theta) \int_{1}^{\infty}\left\{\mathrm{e}^{-\Phi(q) y} W^{(q)}(x)-W^{(q)}(x-y)\right\} \mathrm{d} y<\infty
\end{aligned}
$$

and

$$
\begin{aligned}
& \mid \int_{0}^{1} \int_{y}^{\infty}(y-\theta) \nu(\mathrm{d} \theta)\left\{\mathrm{e}^{-\Phi(q) y} W^{(q)}(x)-W^{(q)}(x-y)\right\} \mathrm{d} y \mid \\
& \leq W^{(q)}(x) \int_{0}^{1} \int_{y}^{\infty} \theta \nu(\mathrm{d} \theta) \mathrm{d} y \\
&=W^{(q)}(x)\left(\int_{1}^{\infty} \theta \nu(\mathrm{d} \theta)+\int_{0}^{1} \theta^{2} \nu(\mathrm{d} \theta)\right)<\infty
\end{aligned}
$$

which proves (13). By a similar reasoning, for $0 \leq x \leq b$, using that

$$
\int_{0}^{\infty} \mathrm{e}^{-q t} \mathbb{P}_{x}\left(U_{t}^{b} \in \mathrm{d} y, t<\sigma^{b}\right) \mathrm{d} t=\frac{W^{(q)}(x)}{W^{(q) \prime}(b)} W^{(q)}(b-\mathrm{d} y)-W^{(q)}(x-y) \mathrm{d} y
$$

(see Theorem 1 (ii) in [28]), we deduce (14]).

Proof of Lemma 4.1. We first show that

$$
\lim _{x \rightarrow \infty} \mathbb{E}_{x}\left[\mathrm{e}^{-q \tau_{0}^{-}} X_{\tau_{0}^{-}} \mathbb{I}_{\left\{\tau_{0}^{-}<\infty\right\}}\right]=0
$$

For this we need some facts about the resolvent measure of a spectrally negative Lévy process. On the negative half line, this measure is given by

$$
\int_{0}^{\infty} \mathrm{e}^{-q t} \mathbb{P}\left(X_{t} \in-\mathrm{d} x\right) \mathrm{d} t=\theta^{(q)}(x) \mathrm{d} x
$$

with

$$
\theta^{(q)}(x)=\Phi^{\prime}(q) \mathrm{e}^{\Phi(q) x}-W^{(q)}(x), \quad x \geq 0,
$$

(see e.g. Corollary 8.9 in [22]). We recall the following two identities derived by Bingham (see Theorem $6 \mathrm{~b}$ in [10]): for $x, q, r \geq 0$,

$$
\begin{aligned}
\mathbb{E}_{x}\left[\mathrm{e}^{-q \tau_{0}^{-}+r X_{\tau_{0}^{-}}}\right. & \left.\mathbb{I}_{\left\{\tau_{0}^{-}<\infty\right\}}\right] \\
& =\frac{q-\psi(r)}{\Phi(q)-r} \theta^{(q)}(x)+(q-\psi(r)) \mathrm{e}^{r x} \int_{x}^{\infty} \mathrm{e}^{-r z} \theta^{(q)}(z) \mathrm{d} z
\end{aligned}
$$

and, for $q>0, r \geq 0$,

$$
\widehat{\theta}(r):=\int_{0}^{\infty} \mathrm{e}^{-r x} \theta^{(q)}(x) \mathrm{d} x=\frac{\Phi^{\prime}(q)}{r-\Phi(q)}-\frac{1}{\psi(r)-q} .
$$

When $r=\Phi(q)$ in $(33)$, the term $\frac{q-\psi(r)}{\Phi(q)-r}$ should be read as $\lim _{r \rightarrow \Phi(q)} \frac{q-\psi(r)}{\Phi(q)-r}=$ $\psi^{\prime}(\Phi(q))=1 / \Phi^{\prime}(q)$. A similar thing is understood when $r=\Phi(q)$ in (34). 
Taking the right-hand derivative in $r$ at zero on both sides of (33) leads to, using the dominated convergence theorem, (13) and (34),

$$
\begin{aligned}
& \mathbb{E}_{x}\left[\mathrm{e}^{-q \tau_{0}^{-}} X_{\tau_{0}^{-}} \mathbb{I}_{\left\{\tau_{0}^{-}<\infty\right\}}\right] \\
& =\frac{\mathrm{d}}{\mathrm{d} r}\left[\frac{q-\psi(r)}{\Phi(q)-r}\right]_{r=0} \theta^{(q)}(x)-\psi^{\prime}(0+) \int_{x}^{\infty} \theta^{(q)}(z) \mathrm{d} z \\
& \quad+q\left(x \int_{x}^{\infty} \theta^{(q)}(z) \mathrm{d} z+\widehat{\theta}^{\prime}(0)+\int_{0}^{x} z \theta^{(q)}(z) \mathrm{d} z\right) .
\end{aligned}
$$

Note that $\left|\widehat{\theta}^{\prime}(0)\right|<\infty$ if and only if $\left|\psi^{\prime}(0+)\right|<\infty$. By $(33)$ and the dominated convergence theorem,

$$
\lim _{x \rightarrow \infty} \theta^{(q)}(x)=\Phi^{\prime}(q) \lim _{x \rightarrow \infty} \mathbb{E}_{x}\left[\mathrm{e}^{-q \tau_{0}^{-}+\Phi(q) X_{\tau_{0}^{-}}} \mathbb{I}_{\left\{\tau_{0}^{-}<\infty\right\}}\right]=0
$$

and, since $\theta^{(q)}(x) \geq 0, \lim _{x \rightarrow \infty} \int_{x}^{\infty} \theta^{(q)}(z) \mathrm{d} z=0$. We now see that 31 holds if

$$
\lim _{x \rightarrow \infty}\left(x \int_{x}^{\infty} \theta^{(q)}(z) \mathrm{d} z+\int_{0}^{x} z \theta^{(q)}(z) \mathrm{d} z\right)=-\widehat{\theta}^{\prime}(0)
$$

In order to show the latter, note that by integration by parts

$$
\int_{0}^{x} z \theta^{(q)}(z) \mathrm{d} z=-x \int_{x}^{\infty} \theta^{(q)}(z) \mathrm{d} z+\int_{0}^{x} \int_{y}^{\infty} \theta^{(q)}(z) \mathrm{d} z \mathrm{~d} y
$$

and by the monotone convergence theorem, Fubini's theorem and L'Hôpital's rule,

$$
\begin{aligned}
\int_{0}^{\infty} \int_{y}^{\infty} \theta^{(q)}(z) \mathrm{d} z \mathrm{~d} y & =\lim _{\lambda \downarrow 0} \int_{0}^{\infty} \mathrm{e}^{-\lambda y} \int_{y}^{\infty} \theta^{(q)}(z) \mathrm{d} z \mathrm{~d} y \\
& =\lim _{\lambda \downarrow 0} \frac{\widehat{\theta}(0)-\widehat{\theta}(\lambda)}{\lambda}=-\widehat{\theta}^{\prime}(0) .
\end{aligned}
$$

Combining (33) with (32) using (34), we deduce

$$
\begin{aligned}
& \mathbb{E}_{x}\left[\mathrm{e}^{-q \tau_{0}^{-}+r X_{\tau_{0}^{-}}} \mathbb{I}_{\left\{\tau_{0}^{-}<\infty\right\}}\right] \\
& \quad=\mathrm{e}^{r x}+(q-\psi(r)) \mathrm{e}^{r x} \int_{0}^{x} \mathrm{e}^{-r z} W^{(q)}(z) \mathrm{d} z-\frac{q-\psi(r)}{\Phi(q)-r} W^{(q)}(x)
\end{aligned}
$$

and by now taking the right-hand derivative with respect to $r$ at zero, we get an expression for the expected discounted deficit at ruin in terms of scale 
functions

$$
\begin{aligned}
\mathbb{E}_{x}\left[\mathrm{e}^{-q \tau_{0}^{-}} X_{\tau_{0}^{-}} \mathbb{I}_{\left\{\tau_{0}^{-}<\infty\right\}}\right] \\
=x-\psi^{\prime}(0+) \int_{0}^{x} W^{(q)}(z) \mathrm{d} z+q \int_{0}^{x}(x-z) W^{(q)}(z) \mathrm{d} z \\
\quad-\frac{\mathrm{d}}{\mathrm{d} r}\left[\frac{q-\psi(r)}{\Phi(q)-r}\right]_{r=0} W^{(q)}(x) \\
=x-\psi^{\prime}(0+) \int_{0}^{x} W^{(q)}(z) \mathrm{d} z+q \int_{0}^{x} W^{(q)}(z) \mathrm{d} z \\
\quad-\frac{q-\Phi(q) \psi^{\prime}(0+)}{\Phi(q)^{2}} W^{(q)}(x),
\end{aligned}
$$

where we have used integration by parts for the second equality. Setting $r=0$ in (35) and using the dominated convergence theorem, we get

$$
\lim _{x \rightarrow \infty}\left(1+q \int_{0}^{x} W^{(q)}(z) \mathrm{d} x-\frac{q}{\Phi(q)} W^{(q)}(x)\right)=0 .
$$

By (31) the right-hand side of (36) goes to zero when $x$ goes to infinity and together with (37) and the continuous differentiability of $W^{(q)}$, we get

$$
\begin{aligned}
\liminf _{x \rightarrow \infty}\left\{K \left(1-\psi^{\prime}(0+) W^{(q)}(x)\right.\right. & \left.+q \bar{W}^{(q)}(x)-\frac{q-\Phi(q) \psi^{\prime}(0+)}{\Phi(q)^{2}} W^{(q) \prime}(x)\right) \\
+ & \left.S\left(q W^{(q)}(x)-\frac{q}{\Phi(q)} W^{(q) \prime}(x)\right)\right\}=0
\end{aligned}
$$

for any $K, S \in \mathbb{R}$. Note that we used here that, for a continuously differentiable function $f$, if we have $\lim _{x \rightarrow \infty} f(x)=a$ with $a \in \mathbb{R}$, then $\liminf _{x \rightarrow \infty} f^{\prime}(x)=0$.

We are now ready to prove the lemma. Using the well-known fact that $\lim _{x \rightarrow \infty} \frac{W^{(q)}(x)}{W^{(q) \prime}(x)}=1 / \Phi(q)$ (see e.g. Section 3.3 in [7]) and L'Hôpital's rule, we deduce

$$
\lim _{y \rightarrow \infty} F(y)=-K \frac{q-\Phi(q) \psi^{\prime}(0+)}{\Phi(q)^{2}}-S \frac{q}{\Phi(q)} .
$$

Since we can write

$$
F(x)=\lim _{y \rightarrow \infty} F(y)+\frac{1-\left[A(x)+\lim _{y \rightarrow \infty} F(y) W^{(q) \prime}(x)\right]}{W^{(q) \prime}(x)}
$$

and $\liminf _{x \rightarrow \infty}\left[A(x)+\lim _{y \rightarrow \infty} F(y) W^{(q) \prime}(x)\right]=0$ by $(38)$, it follows that for all $R>0$ there exists $x \geq R$ such that $F(x)>\lim _{y \rightarrow \infty} F(y)$. Hence $F$ is not ultimately increasing. 
Proof of Lemma 5.2. The first part is proved in Lemma 4 of [7] (see also [9]) when $X$ has paths of bounded variation or $\sigma>0$ and in Lemma 4.2 of [23] when $X$ has paths of unbounded variation and $\sigma=0$. In a similar way, the second part, after a standard application of Itô's formula (note that $x \mapsto S+\int_{0}^{x} A(y) \mathrm{d} y$ is twice continuously differentiable on $\left.(0, \infty)\right)$, follows from (36) and the martingale property of the process $\left(M_{t}\right)_{t \geq 0}$ given by

$$
M_{t}=\mathrm{e}^{-q\left(t \wedge \tau_{0}^{-}\right)} \mathbb{E}_{X_{t \wedge \tau_{0}^{-}}}\left[\mathrm{e}^{-q \tau_{0}^{-}} X_{\tau_{0}^{-}} \mathbb{I}_{\left\{\tau_{0}^{-}<\infty\right\}}\right] .
$$

In order to show the latter, note that by the strong Markov property, we have that

$$
\begin{aligned}
\mathbb{E}_{x} & {\left[\mathrm{e}^{-q \tau_{0}^{-}} X_{\tau_{0}^{-}} \mathbb{I}_{\left\{\tau_{0}^{-}<\infty\right\}} \mid \mathcal{F}_{t}\right] } \\
& =\mathbb{E}_{x}\left[\mathrm{e}^{-q \tau_{0}^{-}} X_{\tau_{0}^{-}} \mathbb{I}_{\left\{\tau_{0}^{-}>t\right\}} \mathbb{I}_{\left\{\tau_{0}^{-}<\infty\right\}} \mid \mathcal{F}_{t}\right]+\mathbb{E}_{x}\left[\mathrm{e}^{-q \tau_{0}^{-}} X_{\tau_{0}^{-}} \mathbb{I}_{\left\{\tau_{0}^{-} \leq t\right\}} \mid \mathcal{F}_{t}\right] \\
& =\mathbb{I}_{\left\{\tau_{0}^{-}>t\right\}} \mathrm{e}^{-q t} \mathbb{E}_{X_{t}}\left[\mathrm{e}^{-q \tau_{0}^{-}} X_{\tau_{0}^{-}} \mathbb{I}_{\left\{\tau_{0}^{-}<\infty\right\}}\right]+\mathbb{I}_{\left\{\tau_{0}^{-} \leq t\right\}} \mathrm{e}^{-q \tau_{0}^{-}} X_{\tau_{0}^{-}} \\
& =\mathbb{I}_{\left\{\tau_{0}^{-}>t\right\}} M_{t}+\mathbb{I}_{\left\{\tau_{0}^{-} \leq t\right\}} \mathrm{e}^{-q\left(t \wedge \tau_{0}^{-}\right)} \mathbb{E}_{X_{\tau_{0}^{-}}}\left[\mathrm{e}^{-q \tau_{0}^{-}} X_{\tau_{0}^{-}} \mathbb{I}_{\left\{\tau_{0}^{-}<\infty\right\}}\right] \\
& =M_{t} .
\end{aligned}
$$

Now $\mathbb{E}_{x}\left|M_{t}\right|<\infty$ follows from the above, (36) and (13) and, in combination with the tower property of conditional expectation, the martingale property of $M$ is shown.

\section{REFERENCES}

[1] H. Albrecher and S. Thonhauser. Optimality results for dividend problems in insurance. RACSAM Rev. R. Acad. Cien., Serie A Mat. 103(2):295-320, 2009.

[2] L. H. R. Alvarez and T. A. Rakkolainen. Optimal payout policy in presence of downside risk. Math. Methods Oper. Res., 69(1):27-58, 2009.

[3] M. Y. An. Logconcavity versus logconvexity: a complete characterization. J. Econom. Theory, 80(2):350-369, 1998.

[4] S. Asmussen. Applied probability and queues. Wiley Series in Probability and Mathematical Statistics: Applied Probability and Statistics. John Wiley \& Sons Ltd., Chichester, 1987.

[5] B. Avanzi. Strategies for dividend distribution: a review. N. Am. Actuar. J., 13(2):217-251, 2009.

[6] F. Avram, A. E. Kyprianou, and M. R. Pistorius. Exit problems for spectrally negative Lévy processes and applications to (Canadized) Russian options. Ann. Appl. Probab., 14(1):215-238, 2004.

[7] F. Avram, Z. Palmowski, and M. R. Pistorius. On the optimal dividend problem for a spectrally negative Lévy process. Ann. Appl. Probab., 17(1):156-180, 2007.

[8] P. Azcue and N. Muler. Optimal reinsurance and dividend distribution policies in the Cramér-Lundberg model. Math. Finance, 15(2):261-308, 2005.

[9] E. Biffis and A. E. Kyprianou. A note on scale functions and the time value of ruin for Lévy insurance risk processes. Insurance Math. Econom., to appear.

[10] N. H. Bingham. Fluctuation theory in continuous time. Adv. in Appl. Probab., $7(4): 705-766,1975$.

[11] E. Boguslavskaya. Optimization problems in financial mathematics: explicit solutions for diffusion models. PhD thesis, University of Amsterdam, 2008. 
[12] T. Chan, A. E. Kyprianou, and M. Savov. Smoothness of scale functions for spectrally negative Lévy processes, 2009. arXiv:0903.1467v1 [math.PR].

[13] N. G. de Bruijn and P. Erdös. On a recursion formula and on some Tauberian theorems. J. Research Nat. Bur. Standards, 50, 1953.

[14] B. de Finetti. Su un' impostazione alternativa dell teoria collettiva del rischio. Transactions of the XVth International Congress of Actuaries, 2:433-443, 1957.

[15] D. C. M. Dickson and H. R. Waters. Some optimal dividends problems. Astin Bull., 34(1):49-74, 2004.

[16] W. Feller. An introduction to probability theory and its applications. Vol. II. Second edition. John Wiley \& Sons Inc., New York, 1971.

[17] H. U. Gerber, X. S. Lin, and H. Yang. A note on the dividends-penalty identity and the optimal dividend barrier. Astin Bull., 36(2):489-503, 2006.

[18] H. U. Gerber and E. S. W. Shiu. On the time value of ruin. N. Am. Actuar. J., 2(1):48-78, 1998

[19] H. U. Gerber, E. S. W. Shiu, and N. Smith. Maximizing dividends without bankruptcy. Astin Bull., 36(1):5-23, 2006.

[20] B. G. Hansen and J. B. G. Frenk. Some monotonicity properties of the delayed renewal function. J. Appl. Probab., 28(4):811-821, 1991.

[21] N. Kulenko and H. Schmidli. Optimal dividend strategies in a Cramér-Lundberg model with capital injections. Insurance Math. Econom., 43(2):270-278, 2008.

[22] A. E. Kyprianou. Introductory lectures on fluctuations of Lévy processes with applications. Universitext. Springer-Verlag, Berlin, 2006.

[23] A. E. Kyprianou, V. Rivero, and R. Song. Convexity and smoothness of scale functions with applications to de Finetti's control problem. J. Theoret. Probab., to appear. arXiv:0801.1951v3 [math.PR].

[24] R. L. Loeffen. Optimality of the barrier strategy in de Finetti's dividend problem for spectrally negative Lévy processes. Ann. Appl. Probab., 18(5):1669-1680, 2008.

[25] R. L. Loeffen. An optimal dividends problem with a terminal value for spectrally negative Lévy processes with a completely monotone jump density. J. Appl. Probab., 46(1):85-98, 2009.

[26] R. L. Loeffen. An optimal dividends problem with transaction costs for spectrally negative Lévy processes. Insurance: Mathematics and Economics, 45(1):41-48, 2009.

[27] A. Müller and D. Stoyan. Comparison methods for stochastic models and risks. Wiley Series in Probability and Statistics. John Wiley \& Sons Ltd., Chichester, 2002.

[28] M. R. Pistorius. On exit and ergodicity of the spectrally one-sided Lévy process reflected at its infimum. J. Theoret. Probab., 17(1):183-220, 2004.

[29] A. Prékopa. On logarithmic concave measures and functions. Acta Sci. Math. (Szeged), 34:335-343, 1973.

[30] P. E. Protter. Stochastic integration and differential equations, volume 21 of Stochastic Modelling and Applied Probability. Springer-Verlag, Berlin, 2005. Second edition. Version 2.1, Corrected third printing.

[31] A. W. Roberts and D. E. Varberg. Convex functions. Academic Press [A subsidiary of Harcourt Brace Jovanovich, Publishers], New York-London, 1973. Pure and Applied Mathematics, Vol. 57.

[32] S. E. Shreve, J. P. Lehoczky, and D. P. Gaver. Optimal consumption for general diffusions with absorbing and reflecting barriers. SIAM J. Control Optim., 22(1):5575,1984 .

[33] S. Thonhauser and H. Albrecher. Dividend maximization under consideration of the time value of ruin. Insurance Math. Econom., 41(1):163-184, 2007.

[34] C. Yin and C. Wang. Optimality of the barrier strategy in de Finetti's dividend problem for spectrally negative Lévy processes: An alternative approach. J. Comput. Appl. Math., 233(2):482-491, 2009. 
Johann Radon Institute for Computational and Applied Mathematics (RICAM), Austrian Academy of Sciences, Altenbergerstrasse 69, A-4040 Linz, Austria

E-mail address: ronnie.loeffen@oeaw.ac.at

Department of Statistics and Actuarial Science, University of Waterloo, 200 University Avenue West, Waterloo (Ontario) N2L 3G1, Canada

E-mail address: jf2renaud@math.uwaterloo.ca 